\title{
Gray-Scale Thinning Algorithm Using Local Min/Max Operations
}

\author{
Kyoung Min Kim ${ }^{1,2}$, Buhm Lee,,* Nam Sup Choi ${ }^{2}$, Gwan Hee Kang², \\ Joong Jo Park ${ }^{1,3}$, and Ching Y. Suen ${ }^{1}$ \\ ${ }^{1}$ Centre for Pattern Recognition and Machine Intelligence (CENPARMI), \\ Concordia University, 1455 de Maisonneuve Blvd. West, Montreal, Canada H3G 1M8 \\ $\{\mathrm{kkm}$, jjpark, suen\}@cenparmi.concordia.ca \\ ${ }^{2}$ Department of Electrical Engineering, Yosu National University, San 96-1, \\ Dundeok-dong, Yeosu-si, Jeollanam-do, 550-749, Korea \\ $\{\mathrm{kkm}$, buhmlee, nschoi\} @yosu.ac.kr \\ ${ }^{3}$ Department of Control and Instrumentation Engineering, Gyeongsang National University, \\ Gajwa-dong, Jinju-si, Gyeongsangnam-do, 660-701, Korea \\ jjpark@nongae.gsnu.ac.kr
}

\begin{abstract}
A gray-scale thinning algorithm based on local min/max operations is newly proposed. Erosion and dilation properties of local $\mathrm{min} / \mathrm{max}$ operations create new ridges from the given image. Thus grey scale skeletons can be effectively obtained by accumulating such ridges. The proposed method is quite salient because it can be also applied to an unsegmented image in which objects are not specified.
\end{abstract}

\section{Introduction}

Thinning is one of the most important pre-processing steps for image analysis and understanding. Many thinning techniques showing quite good results have been developed for binary image [1][2][3]. However, as the generalization of bi-level thinning, the grey level thinning has been not yet actively studied, even though considerable its own particular applications such as thinning the object of non-uniform brightness or determining the tinned edge are exist.

As a previous study on grey-level thinning, Peleg proposed the method that uses the erosion and dilation properties derived from the local min/max operation [4]. This method has some merits that a pre-processing to divide a given grey image into the object and background is not required and the extracted edge position usually correspond to the part having large pixel value in the object. However, it shows poor edge connectivity.

Salari proposed a thinning technique based on ridge tracing in which the skeleton of an object is obtained by detecting the ridge parts resulted from erosion processing [5]. Skeleton with single thickness can be obtained and its connectivity is also guaranteed, although it has a restriction that the object in image should be separated in advance. Wang proposed another approach using the connectivity number that thins a

* Corresponding author. 
grey image by replacing each pixel value to the minimum value nearby [6]. This method can perform thinning for the object not separated from background. However, in this method some skeletons can be lost and the connectivity is not guaranteed because the connectivity number cannot provide complete information about some feature points like end, node, or crossing points.

In this paper, we proposed a new grayscale thinning method based on local $\mathrm{min} / \mathrm{max}$ operation. Unlike Salari or Wang's method where the ridges created by eroding only the pixels satisfying some specific conditions are determined as the final skeletons, the proposed method extracts the skeletons by repeatedly carrying out the procedure that detects the ridges newly created by local min operation and then recover the connectivity of disconnected skeleton using connectivity information, after detecting the already existing ridges using the local $\mathrm{min} / \mathrm{max}$ operation.

The novelty of our approach lies in the fact that the connectivity of skeleton is well reserved, its position corresponds to the part having large pixel value, and separating the object from background is not required.

\section{Principles of Greyscale Thinning Algorithm Through Ridge Detection}

This chapter will describe the basic principles of the grayscale thinning algorithm being proposed in this research. The ridges detected through grayscale thinning should be as thin as 1 pixel width, have continuous characteristics and should have the highest pixel values within an object. Also, because it is usually difficult to clearly separate objects from the background in grayscale images, a thinning algorithm that can detect ridges even without separation would be appropriate.

One thinning algorithm that can satisfy those conditions is to gradually erode the image until ridges become detectable, and then finally obtaining a skeleton. Fig.1 demonstrates this process in one-dimensional diagram.

First Fig.1(a) shows side-cut of original image, and target skeletons in this image would be located in (1), (2) and (3). Performing ridge detection on this image will only find (1) as a ridge because (1) is already shaped like a ridge but (2) and (3) are mostly flat. Fig.1(b) is eroded image of 1(a) with the previously extracted ridge restored on top. Performing ridge detection on this image will find (1) and (3) since (1) is already a ridge, (3) just became a ridge through the erosion process, and (2) still remains flat. Fig. 1(c) is further eroded image of 1(b) with ridges detected in 1(b) restored. In this case (2) now becomes a ridge and thus another ridge detection process applied will find all of (1), (2) and (3) as ridges. Fig.1(d) shows above erosion detection process repeated several times with the ridges restored. This image will not change any more with further processing. Performing ridge detection on this final image and taking the detected ridge values obtains Fig.1(e). This is the final result of grayscale thinning algorithm and corresponds to the skeleton of the original image.

If the background's grayscale is needed to be kept in order to obtain some other information, Fig.1(d) would be a suitable result. The processing mechanism needed in these grayscale thinning are erosion computation and ridge detection methods. This paper proposes a grayscale thinning algorithm that finds skeletons through performing local $\mathrm{min} / \mathrm{max}$ operations. 


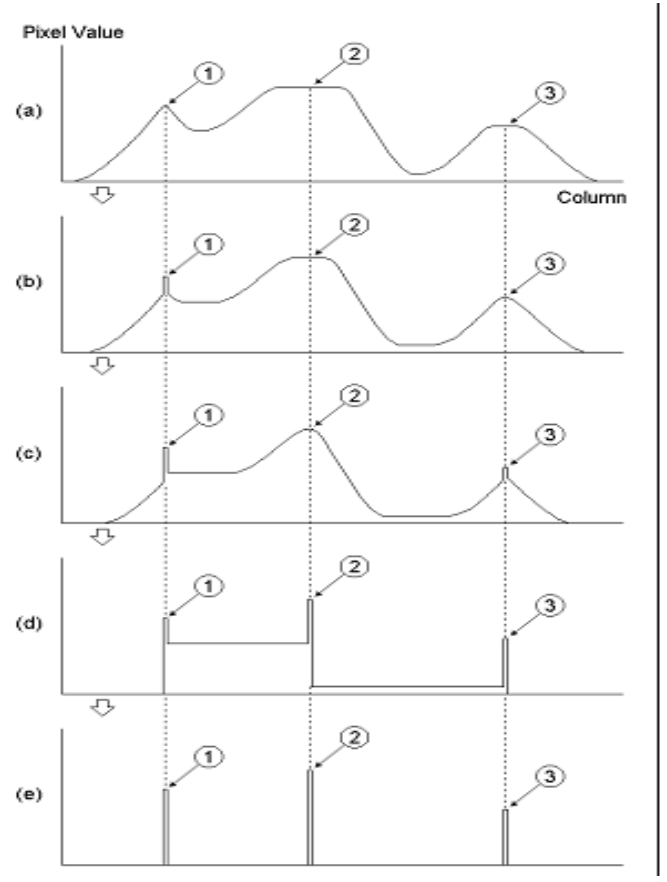

Fig. 1. Process of gray level thinning by proposed algorithm (a) Original image, (b), (c), (d) Process of image erosion and ridge detection, (e) Thinned image

\section{The Proposed Gray-Level Thinning Algorithm}

Basically, the proposed method needs the erosion operation and ridge detection techniques [7][8]. We employ local $\mathrm{min} / \mathrm{max}$ operation to implement these techniques. As a parallel neighborhood operation, the local min operation used for image erosion and local max for dilation replace each pixel value of a given image with the maximum and minimum value among itself and its neighbors, respectively. Let $\Pi_{\phi}=\left\{\mu_{\phi}^{i j} ; i=1,2, \ldots, I, j=1,2, \ldots, J\right\}$ be the $I \times J$, grey-level image array. Then the erosion operation for a pixel at $(\mathrm{I}, \mathrm{J})$ is mathematically written as

$$
\begin{gathered}
\operatorname{EROSION}\left\{\mu_{\phi}^{i j}\right\}=\min _{R}\left\{\mu_{\phi}^{i j}\right\} \\
\prod_{\text {ERosson }} \doteq \operatorname{EROSION}\left(\prod_{G}\right)=\min _{R}(\Pi)
\end{gathered}
$$

where, $\min _{R}$ is the notation for the local min operator over the region $\mathrm{R}$.

Then, the positions of ridge pixels in the image can be detected by calculating the difference between the original image $\Pi_{G}$ and its opened version obtained by the morphological opening operation, i.e., the ridge image $\Pi_{R I D G E}$ is obtained by 


$$
\Pi_{R I D G E}=\left\{\mu_{R I D G E}^{i j} ; i=1,2, \ldots, I, j=1,2, \ldots, J\right\} \quad 0 \leq \mu_{R I D G E}^{i j} \leq 1
$$

where $\quad \mu_{R I D G E}{ }^{i j}=\left\{\begin{array}{ccc}\mu_{G}^{i j} & \text { if } \mu_{G}^{i j}-\max _{R}\left\{\min _{R}\left\{\mu_{G}^{i j}\right\}\right\}>0, \\ 0 & \text { otherwise }\end{array}\right.$

In $\Pi_{R I D G E}$, even noise-like ridges can be considerably included, since all possible ridges are detected by eq. (3). The clearer ridge, which has relatively large pixel value when compared to its neighborhood, can be detected by slightly modifying this equation as follows:

$$
\mu_{R I D G E_{-} n}{ }^{i j}=\left\{\begin{array}{ccc}
\mu_{G}^{i j} & \text { if } & \mu_{G}^{i j}-\max _{R}{ }^{(1)}\left\{\min _{R}{ }^{(1)}\left\{\mu_{G}{ }^{i j}\right\}\right\}>0 \& \\
& & \mu_{G}^{i j}-\max _{R}{ }^{(2)}\left\{\min _{R}{ }^{(2)}\left\{\mu_{G}{ }^{i j}\right\}\right\}>\text { Height } \\
0 & \text { otherwise }
\end{array}\right.
$$

where the superscripts (1) and (2) on operation $\min _{R}$ or $\max _{R}$ denote the number of iterations of these operations applied to the image. The higher the value of threshold, Height is assigned, the larger the pixel values of ridges can be obtained. Then the composite image, $\Pi_{C O M P n}$ is obtained by using $\Pi_{R I D G E}$ and $\Pi_{\text {EROSION }}$ as follows:

$$
\Pi_{\text {СОМP }}=\left\{\mu_{\text {COMP }}^{i j} ; i=1,2, \ldots, I, \quad j=1,2, \ldots, J\right\}
$$

where, $\mu_{\text {COMP }}^{i j}=\max _{p}\left(\mu_{R I D G E}^{i j}, \mu_{\text {EROSION }}^{i j}\right), \mu_{R I D G E}^{i j} \in \Pi_{R I D G E}$, and $\mu_{\text {EROSION }}^{i j} \in \Pi_{\text {EROSION }}$. Plus, $\max _{\mathrm{P}}()$ denotes the local max operator applied to each pixel on two images, $\Pi_{\text {RIDGE }}$ and $\Pi_{\text {EROSION }}$.

The above ridge detection and erosion processes are repeated using the obtained $\Pi_{\text {СОMP }}$ as input image again. During this process the erosion operation is what makes ridges visible, and ridge detection is what finds the skeleton. According to this method, the ridges found during the process are part of the final skeleton, and thus newly found ridges through eq.(3) are accumulated on existing ridges using eq.(5). Ridge detection is then repeated to build up the final skeleton. This process is iterated until $\Pi_{C O M P}$ image does not change any further. At this stage ridge detection is applied for one last time and ridges found here are determined to be the final skeleton of the image.

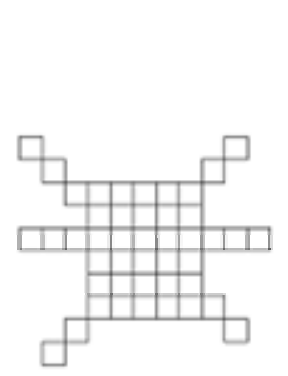

(a)

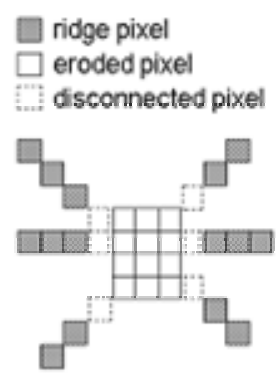

(b)

Fig. 2. Connectivity problem: (a) Original image (b) Composite image $\left(\Pi_{\text {СOMP }}\right)$ 
However, this method of grayscale thinning will create a connectivity problem between the already obtained ridges and eroded image in terms of pixel values. Fig. 2 shows this problem. Fig.2(b) is the $\Pi_{\text {СOMP }}$ image after one cycle of above process on Fig.2(a). This connectivity problem in $\Pi_{\text {СOMP }}$ image should be resolved.

This disconnection can be restored using grayscale connectivity number information. Grayscale connectivity number is an extension of 8-connectivity number concept for application on grayscale images. The connectivity number $\mathrm{C}_{8}\left(\mathrm{x}_{0}\right)$ of central pixel $\mathrm{x}_{0}$ on $3 \times 3$ image area is computed as the number of changes of 8 pixel values $f_{k}$, as defined in following equation (6) and (7), from -1 to 1 . Pixel scanning is done by one clockwise rotation (i.e. $\mathrm{f}_{8}, \mathrm{f}_{7}, \ldots, \mathrm{f}_{1}$ ), ignoring 0 values and taking $\mathrm{x}_{9}=\mathrm{x}_{1}$. Figure 3 shows $\mathrm{x}_{0}$ and distribution of obtained $\mathrm{f}_{\mathrm{k}}$.

$$
\begin{aligned}
& f_{2 l-1}=-1 \text {, if } \mathrm{x}_{2 l-1}<\mathrm{x}_{0} \text { and }\left(\mathrm{x}_{2 l-1}<\mathrm{x}_{2 l} \text { or } \mathrm{x}_{2 l-1}<\mathrm{x}_{2 l+1}\right) \\
& 1 \text {, if } \mathrm{x}_{2 l-1}>\mathrm{x}_{2 l+1} \text { and } \mathrm{x}_{2 l+1}<\mathrm{x}_{0} \\
& 0 \text {, otherwise } \\
& f_{2 l-1}=\left\{\begin{array}{ccc}
1 & \text { if } & \mathrm{x}_{2 l}>\mathrm{x}_{2 l+1} \text { and } \mathrm{x}_{2 l+1}<\mathrm{x}_{0} \\
0 & \text { otherwise }
\end{array} \quad(\mathrm{l}=1,2,3,4)\right.
\end{aligned}
$$

\begin{tabular}{|l|l|l|}
\hline $\mathrm{f}_{4}$ & $\mathrm{f}_{3}$ & $\mathrm{f}_{2}$ \\
\hline \hline $\mathrm{f}_{5}$ & $\mathrm{x}_{0}$ & $\mathrm{f}_{1}$ \\
\hline \hline $\mathrm{f}_{6}$ & $\mathrm{f}_{7}$ & $\mathrm{f}_{8}$ \\
\hline
\end{tabular}

Fig. 3. pixel $x_{0}$ and $f_{k}$

However the grayscale connectivity number found with this method does not completely provide connectivity status information as contrast to connectivity numbers on binary images. Experiment using grayscale connectivity number found through above method shows that although connected points are correctly detected from the obtained information, detection is not guaranteed on end, node and crossing points. In fact, pixel that was calculated with above method to have value of 2 is guaranteed to be a connectivity point, but end, node and crossing points are not guaranteed to be consistent with values such as 1,3 , and 4 . This grayscale thinning algorithm, however, effectively detects end, node and crossing points during the ridge detection process. Therefore only the connectivity points are required to keep connectivity of the skeleton, and the grayscale connectivity numbers may be used effectively.

This algorithm uses following connectivity restoration method: It scans only pixels that are not part of a ridge among all pixels of composite image $\Pi_{C O M P}$ at current stage. The pixel $\mathrm{x}_{0}$ being scanned is changed to the corresponding pixel value at previous stage composite image, while its 8-connectivity values keep their pixel values of current stage composite image. Then the grayscale connectivity number $\mathrm{C}_{8}\left(\mathrm{x}_{0}\right)$ is computed from above data. If the connectivity number found is 2 or greater, the scanned pixel's value is changed to the corresponding pixel value of previous stage composite image. The connectivity of skeleton is then restored, based on the characteristic that pixel values of grayscale image do not change dramatically about its neighboring val- 
ues. This modified composite image is used again as input image and through iterated ridge detection and erosion process a continuous skeleton can be obtained.

The number of iterations required on this algorithm is automatically determined. However a same final skeleton can be obtained by number of iteration corresponding to $1 / 2$ of maximum ridge width of an object in the image. Therefore if the maximum ridge width of an object in image can be obtained beforehand, number of iteration can be determined before and shorten the required processing time.

\section{Experiments and Results}

This experiment uses artificial pattern images, character images taken with camera and fingerprint image taken from scanner, all of which are 256-scale grey images. The algorithm's local min/max operations used in erosion and ridge detection uses 4neighbour as neighboring area. Fig.4 shows experimental results on an artificial 256x190 size pattern image. Since Peleg's method does not recognize connectivity, the upper right and middle pattern shows connectivity problem. Salari's method fails to find proper skeleton on upper right pattern. His method erodes outlines of the object within image relative to the background pixel value, and so erosion can't be performed, and thus the skeleton can't be found, if there is a grayscale distribution shaped as a well within the object that has only little higher pixel values than the background. Wang's method shows problems in connectivity and skeleton shape in upper right pattern and middle pattern. This is because the grayscale connectivity numbers used in this method does not provide sufficient information. In contrast, our proposed method shows much better performance in both connectivity and skeleton shape. One issue is the appearance of unnecessary branches in left pattern, and this happens because proposed method first extract ridges then erodes the new image again to find additional ridges. Peleg and Wang's method also shows similar artifacts in their results. This result obtained final ridges with the value of height set to 30 in eq. (4).

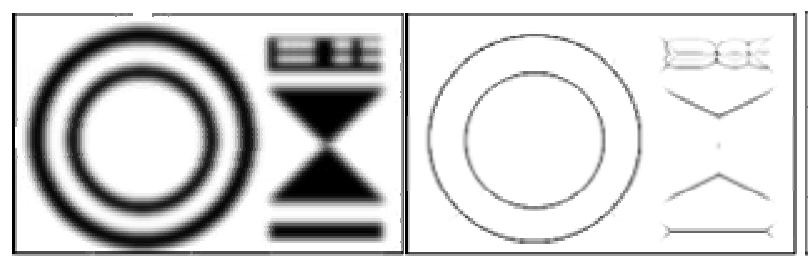

(a)

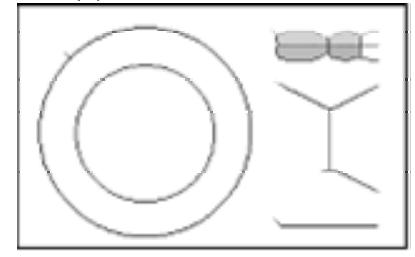

(d)

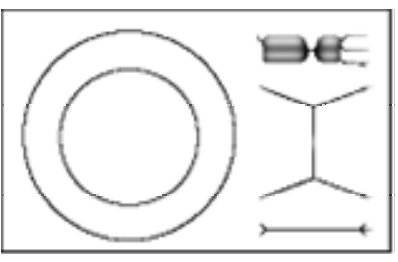

(c)

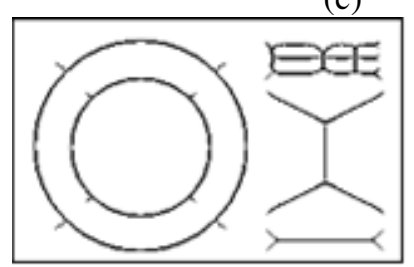

(e)

Fig. 4. Thinning results on the pattern image: (a) Original image, (b) Peleg, (c) Salari, (d) Wang, (e) Proposed algorithm 
Fig.5 shows experimental results on $256 \times 256$ size fingerprint image obtained through thresholding. Peleg's method is result of thresholding with threshold value of 20 , and shows problems in both skeleton shapes and connectivity because it does not consider connectivity in the first place. Salari's method fails to find some skeletons by same reasons on Fig.4. Wang's method shows imperfect connectivity due to its

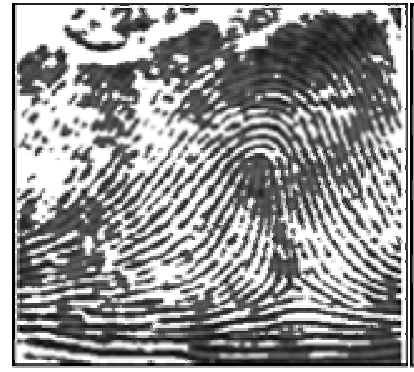

(a)

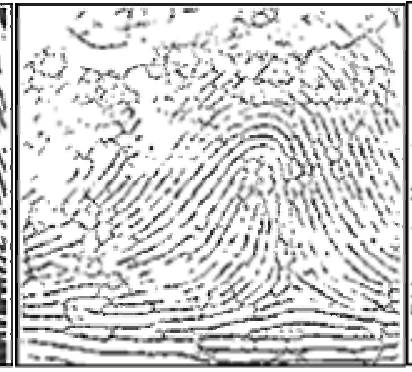

(b)

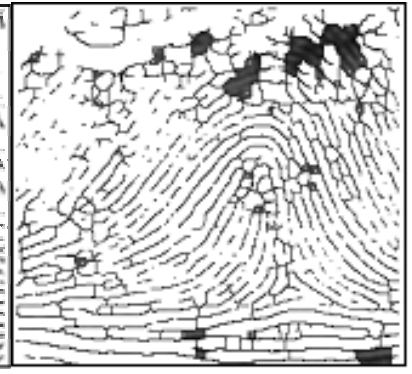

(c)

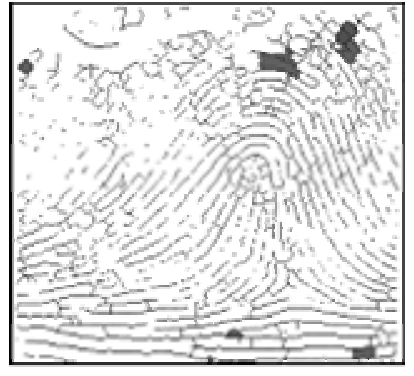

(d)

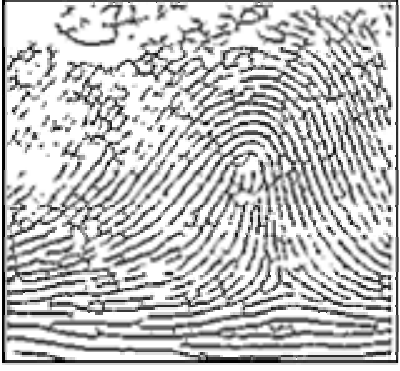

(e)

Fig. 5. Thinning results on the segmented finger print image: (a) Original image, (b) Peleg algorithm, (c) Salari algorithm, (d) Wang algorithm, (e) Proposed algorithm

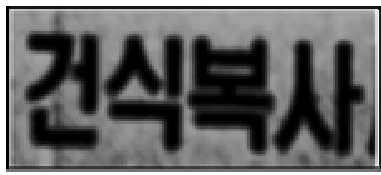

(a)

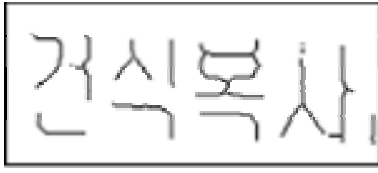

(d)

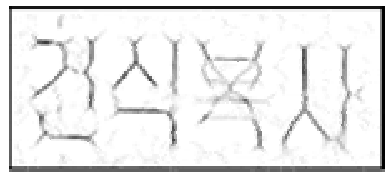

(b)

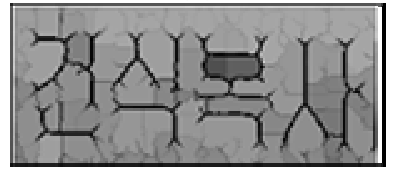

(e)

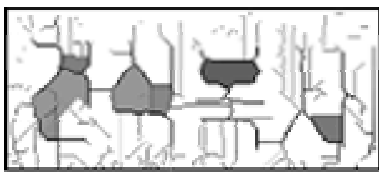

(c)

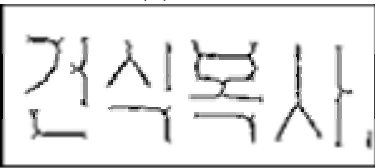

(f)

Fig. 6. Thinning results on Korean character image: (a) Original image, (b) Peleg algorithm, (c) Wang algorithm, (d) Wang algorithm(T=220), (e) Proposed algorithm, (f) Proposed algorithm( $\mathrm{T}=240)$ 
grayscale connectivity numbers. In contrast the proposed method shows much better performance in connectivity and skeleton formation. This result obtained final ridges using height set to 20 in eq. (4).

Fig.6 shows experimental results on image containing characters that are not clearly separated. Salari's method can't be applied in this kind of images. Fig.6(b) is result of Peleg's method, and 6(c) is Wang's method, both performed on original image 6(a). Fig.6(d) is result of thresholding applied on 6(c) with threshold value of 220. Fig. 6(e) is image just before obtaining final ridges using proposed method, and 6(f) is result of thesholding with threshold value of 240. It can be observed that the proposed method provides better results again in terms of skeleton shape and connectivity.

To summarize above experiments: Peleg's method is an early thinning method using local $\mathrm{min} / \max$ operation that shows connectivity problems. Salari's method can't be applied at all if objects within image aren't completely separated, and fails to perform thinning on circular patterns with different grayscale value than the background. Finally although Wang's method provide better skeleton compared to other methods but still poses connectivity issues. Our proposed method however shows improved characteristics in terms of skeleton shape and connectivity. The skeleton obtained from proposed method has width of around 2 pixels; this may be resolved by applying additional binary image thinning algorithm on final image, but further research seems necessary to obtain 1-pixel wide skeleton.

\section{Conclusion}

This research proposed a grayscale image thinning method using erosion and dilation functions of local $\mathrm{min} / \mathrm{max}$ operations. This method extracts skeleton image of grey image using image erosion and ridge detection. The erosion process makes new ridges appear, and then ridge detection is used to extract the skeleton. Although this method uses local $\mathrm{min} / \mathrm{max}$ operations used in existing Peleg's method it differs in concept of ridge detection and skeleton extraction methods. It is also different than Salari method since ridge is directly extracted and is not dependent on object's outline. This method also uses application of grey connectivity numbers Wang conditionally used to remove pixels to restore connectivity on skeleton.

This method preserves connectivity of obtained skeleton. It is located on the largest pixel value areas in the object, and the method does not require clear separation of objects within image. The major mathematical operations used in this method are simple parallel-processing local $\mathrm{min} / \mathrm{max}$ operations which reduces processing time required on hardware. Experimental results show much improved performance in skeleton shapes and connectivity when compared with other existing methods.

\section{References}

1. L. Lam, S. W. Lee and C. Y. Suen,: Thinning Methodologies - A Comprehensive Survey. IEEE Trans. Pattern Analysis and Machine Intelligence, 14 (1992) 869-885

2. V. K. Govindan and A. P. Shivaprasad: A pattern adaptive thinning algorithm, Pattern Recognition, 20 (1987) 623-637 
3. T. Y. Zhang and C. Y. Suen: A fast parallel algorithm for thinning digital patterns, Comm. of the ACM, 27 (1984) 236-239

4. S. Peleg and A. Rosenfeld: A min-max medial axis transformation, IEEE Trans. Pattern Analysis and Machine Intelligence, 3 (1981) 208-210

5. E. Salari and P. Sly: The Ridge-Seeking Method for Obtaining the Skeleton of Digital Images, IEEE Trans. System, Man, and Cybernetics, 14 (1984) 524-528

6. C. Wang and K. ABE: A Method of Gray-scale Image Thinning : The Case without Region specification for Thinning, IEEE 11th Int. Conf. on Pattern Recognition, 3 (1992) 404-407

7. Y. Nakagawa and A. Rosenfeld: A note on the use of local min and max operations in digital picture processing, IEEE Trans. System, Man, and Cybernetics, 8 (1978) 632-635

8. K. M. Kim, J. J. Park, M. H. Song I. C. Kim and C. Y. Suen: Detection of ridges and ravines using fuzzy logic operations, Pattern Recognition Letters, 25 (2004) 743-751 